\title{
Anabases
}

ANABASES Traditions et réceptions de l'Antiquité

$7 \mid 2008$

Varia

\section{Péplum de Blutch, un Satiricon postmoderne}

\section{Vivien Bessières}

\section{OpenEdition}

Journals

Édition électronique

URL : http://journals.openedition.org/anabases/2542

DOI : 10.4000/anabases. 2542

ISSN : 2256-9421

\section{Éditeur}

E.R.A.S.M.E.

\section{Édition imprimée}

Date de publication : 1 mars 2008

Pagination : 257-262

ISSN : 1774-4296

\section{Référence électronique}

Vivien Bessières, «Péplum de Blutch, un Satiricon postmoderne », Anabases [En ligne], 7 | 2008, mis en ligne le 01 décembre 2011, consulté le 21 octobre 2019. URL : http://journals.openedition.org/ anabases/2542 : DOI : 10.4000/anabases. 2542

Ce document a été généré automatiquement le 21 octobre 2019

(c) Anabases 


\title{
Péplum de Blutch, un Satiricon postmoderne
}

\author{
Vivien Bessières
}

1 Les années 90 ont vu l'émergence dans les pays francophones de ce qu'on a appelé, depuis, la «nouvelle bande dessinée ». Cette nouvelle vague, que le septième art avait connue, mais qui manquait au neuvième, a en partie rompu avec les genres populaires, l'aventure et les gags, les séries commerciales et les formats traditionnels. Elle a privilégié la forme du "roman graphique ${ }^{1}$ ». Prenant acte du premier renouveau des années 70, elle a fait dans l'autobiographie, le journal intime, le carnet de voyage, le reportage et, notamment à travers les œuvres de l'Oubapo («Ouvroir de bande dessinée potentielle», fondé en 1992 par des auteurs de la maison d'édition L'Association), l'expérimentation poétique et picturale. Mais la rupture s'est accompagnée d'un retour au second degré des genres et des séries : histoires de pirates, polar, heroïc fantasy, western, tout y est passé, revu et corrigé, et, dans le lot, la bande dessinée antiquisante. Cette dernière était pourtant connotée. Elle renvoyait au réalisme un peu vieille école d'Alix, à l'humour gaulois d'Astérix, à des productions pédagogiques comme Alcibiade Didascaux ou à des séries de pur divertissement parues dans le sillage du péplum italien. Rien là qui ait pu séduire a priori les jeunes auteurs. Alors, pourquoi ? était-ce le pari de réinvestir un champ abandonné au kitsch ? la volonté de se frotter à une matière qui donnerait ses lettres de noblesse au neuvième art? Parmi les titres antiquisants de cette "nouvelle bande dessinée ", on pourrait citer, pêle-mêle, Faune d'Aristophane, paru en 1995, Gilgamesh de Franz Duchazeau et Gwen de Bonneval, paru en 2003, Priape de Nicolas Presl, paru en 2006, et toute la veine antique du productif Joann Sfar ${ }^{2}$. C'est cependant sur Péplum que nous nous pencherons, œuvre majeure de Blutch, publiée en 1996 par la maison indépendante Cornélius. L'album se présentant comme une libre adaptation du Satiricon de Pétrone; nous étudierons en particulier la manière dont cet intertexte fonctionne.

2 L'intrigue de ce roman graphique est très différente de celle du roman latin. Des exilés romains découvrent «aux frontières de l'Empire » une femme très belle emprisonnée dans un bloc de glace. Ils décident de l'emporter. Le héros, pour en profiter seul, tue 
son compagnon, un patricien romain nommé Publius Cimber, et se fait passer pour lui. Pendant ce temps, à la Curie, Metellus Cimber implore César pour qu'il ordonne le retour d'exil de son frère Publius Cimber. César refuse et meurt sous le poignard des conjurés des Ides de mars. Retour au héros : il rencontre un jeune garçon dont il tombe amoureux. Tous deux sont faits prisonniers par un général romain. Ce dernier découvre que le héros a usurpé le nom de Publius Cimber, mais, fasciné par la beauté du jeune garçon, il leur laisse la vie sauve. Le héros retrouve la femme glacée. Il part avec elle en laissant son "petit frère " avec le général. Il rencontre une actrice dont il tombe amoureux, mais devient impuissant. Une sorcière intervient pour le soigner : rien n'y fait. Dans un musée, le héros désespéré rencontre un poète qui le frappe pour s'emparer de la femme gelée. Il s'embarque de nouveau, fait naufrage et échoue sur une plage, où une femme en furie court sur lui pour le tuer. Il se défend et finit par l'égorger. Des gens arrivent pour l'acclamer et le couronner : ils avaient organisé le combat. On le mène dans une maison où est entreposée la femme glacée, qui bien qu'elle ait dégelé, reste inerte.

3 Le lecteur du roman de Pétrone aura reconnu quelques figures : le héros Encolpe, son mignon Giton, le poète Eumolpe... Il aura retrouvé des scènes, comme celles du musée et de la navigation. Toutefois, dans l'économie générale du récit, une seule séquence est directement inspirée du Satiricon, celle de l'impuissance du héros arrivé à Crotone. Comme on lui demandait: "Ton Péplum est-il une adaptation du livre de Pétrone ou s'en inspire-t-il librement?», Blutch répondait: "C'est comme une suite. Quand on aime un livre, on n'a jamais envie qu'il finisse. [...] Pour Péplum, je me suis présomptueusement arrogé le droit de continuer le récit de Pétrone que j'adore depuis des années. J'ai été aidé par le fait qu'il s'agisse d'un roman inachevé, une sorte de vestige $^{3}$.»Continuation, donc, plus qu'adaptation. Cette piste de lecture est séduisante, d'autant que l'épisode de Crotone appartient à ces fragments ultimes du roman, mal rattachés à ce qui les précède. On se dit que Blutch s'est proposé en quelque sorte de combler les lacunes, de restaurer une partie de la ruine antique.

4 L'explication ne semble toutefois pas suffire. Après tout, aucun des noms du roman n'est conservé et la scène de l'impuissance elle-même est sensiblement modifiée : la Circé du roman devient une actrice jouant Ariane. De plus, d'autres intertextes viennent parasiter cette supposée continuation. Citons, entre autres : la figure de la femme glacée, empruntée à un ballet de Roland Petit ${ }^{4}$, la représentation des Ides de Mars, reprise mot pour mot d'une scène du Jules César de Shakespeare ${ }^{5}$, diverses situations inspirées du film de Fellini, et non du roman de Pétrone, etc. Harold Bloom, dans Anxiety of influence, envisageait la catégorie intertextuelle du clinamen: un texte qui continue, en le déviant, un texte antérieur, qui se greffe à ce dernier tout en lui faisant opérer un écart ${ }^{6}$. C'est bien cette catégorie que semble illustrer Péplum, faisant le tri dans le roman, poursuivant les fragments en privilégiant certaines caractéristiques et en en gommant d'autres. D'un point de vue narratif, Blutch opère un double déplacement.

5 a. Tout d'abord, il déplace le projecteur du festin de Trimalcion vers sa périphérie. Le festin est le passage le plus connu, commenté, admiré du roman. Or Péplum n'emprunte quasiment rien à cette scène, mais réécrit longuement l'épisode de Crotone, le dernier, beaucoup moins connu. Pourquoi? Florence Dupont et Thierry Éloi opposaient l'espace-temps du festin, obscène, anti-érotique, satirique, aux espaces-temps collatéraux où, notamment, Encolpe et Giton, le héros et son amant, se retrouvent tous 
les deux dans un contexte amoureux ${ }^{7}$. Lors du festin, toute l'érotique qui entoure les protagonistes est annulée, rendue impossible: les deux jeunes hommes libres ne peuvent s'adonner au bel amour pédérastique dans un univers de renversement obscène où tout est mélangé, où un affranchi peut insulter un homme libre, où le puer delicatus est un vieux garçon aux yeux chassieux, où l'on parfume les pieds des convives, c'est-à-dire où le bas corporel devient le haut. Blutch se tourne vers des épisodes moins parodiques et ironiques, où l'amour d'Encolpe peut s'érotiser.

b. Ensuite, l'auteur déplace l'intrigue dans le temps. L'inscription de la scène shakespearienne situe l'histoire à la fin de la République, et non à l'heure de la décadence impériale. L'idée d'une décadence de l'Empire sous Néron ou Caligula est certes discutable: le discours sur la dissolution des mœurs est une constante romaine, qui apparaît dès la «belle époque » de la République. Mais ce sont les représentations qui ici importent: Blutch, changeant de temps, change l'esprit de l'histoire et efface largement les manifestations obscènes. «Dans la littérature de la fin du xix siècle, on s'est beaucoup intéressé à la décadence de Rome, au côté sulfureux. Moi, je voulais faire quelque chose plutôt à la fin de la République et au début de l'Empire, à l'époque du second Triumvirat ${ }^{8}$. $»$ L'auteur ne fait pas un énième péplum sur la décadence. Ce qu'il retient de Pétrone c'est l'étrangeté, l'artificialité, pas l'obscénité ni la peinture orgiaque.

7 Mais Péplum ne s'est pas contenté de démarquer le récit de Pétrone. Il fait un usage très particulier du texte même du Satiricon, à travers l'emploi postmoderne de l'«implicitation ». L'impli-citation est un terme inventé par Bernard Magné à propos de La Vie mode d'emploi de Georges Perec ${ }^{9}$. Ce dernier a inséré clandestinement dans le cours de son œuvre des citations d'autres auteurs, sans les démarquer par des guillemets ni en révéler la provenance. Il faut attendre les «Pièces annexes » en fin de roman pour connaître la liste des œuvres plagiées. Dans Péplum, la plus grande partie du texte est constituée de ce genre d'impli-citations. Comme chez Perec, on sait seulement à la dernière page de l'album que «Péplum est librement inspiré du Satiricon de Pétrone ». Or l'adverbe « librement » a ici tout son sens. Les citations sont déplacées, insérées dans d'autres situations, détournées de leur signification, selon divers types de décalages.

a. Des décalages de contextes. Au début de l'album, devant la femme glacée, le héros se demande : «Est-elle réellement vivante sous son manteau de glace ? » Et un compagnon répond : «À coup sûr ! Ses yeux sont plus brillants que des étoiles scintillant par une nuit sans lune. Une fois dégelée, nous en tirerons une fortune au marché ${ }^{10}$ ! Dans le roman, Encolpe rencontre une femme dont il fait le portrait. Voici ce qu'il dit dans la traduction de Pierre Grimal ${ }^{11}$, celle qu'a manifestement choisie Blutch: "Ses yeux étaient plus brillants que des étoiles scintillant par une nuit sans lune ${ }^{12}$. $\gg$ La phrase de Pétrone appartient à une sorte de blason décrivant de la tête aux pieds l'objet du coup de foudre : cheveux, front, sourcils, yeux, narines, lèvres, menton, cou, mains, pieds. Blutch ne prélève du portrait qu'une partie et poursuit sur une remarque cupide du personnage: «Nous en tirerons une fortune au marché! » C'est le décalage entre l'envolée poétique sur les yeux et la remarque triviale qui fait ici sens. Blutch ne se soucie pas de cohérence psychologique. Il développe une poétique du discontinu: les personnages sont des puzzles de citations disparates produisant étrangeté et poésie.

9 b. Des décalages de tonalités. Le général romain de Péplum est tombé amoureux de l'amant du héros. Il s'assied auprès du jeune homme allongé sur son lit et dit : « Dame Vénus, si je puis donner un baiser à cet enfant, demain je lui apporterai une paire de colombes ${ }^{13}$ 
... » Dans le Satiricon, le poète Eumolpe fait le récit d'une de ses aventures ${ }^{14}$. Tombé amoureux d'un jeune enfant de bonne famille, il avait trouvé un stratagème pour obtenir de lui le fruit de ses désirs. Auprès du garçon qui faisait semblant de dormir, il formulait un vœu : «Dame Vénus, si je puis donner un baiser à cet enfant sans qu'il s'en aperçoive, demain je lui apporterai une paire de colombes... » Le jeune garçon pouvait ainsi accepter de se donner à Eumolpe sans pour autant perdre la face : il faisait croire qu'il dormait, mais restait disponible pour le poète et n'en attendait pas moins sa récompense. Eumolpe, au cours des nuits suivantes, promettra des cadeaux de plus en plus exorbitants. Blutch a ôté de son collage la proposition à tonalité comique, que nous avons mise en italiques. De plus, il n'a retenu qu'un élément de la surenchère humoristique, et le plus céleste : «la paire de colombes » (les cadeaux suivants étant des coqs puis des trotteurs). Il ne s'agit plus d'un conte grivois. On passe chez Blutch à une tonalité lyrique. Il n'est pas jusqu'au rythme qui ne soit poétique : 4 / 12 / 2 / 12 (le " sans qu'il s'en aperçoive » aurait d'ailleurs brisé la cadence).

c. Des décalages de genres. La planche montre des matelots attrapant des mouettes. Blutch légende: «Le chœur des soldats ${ }^{15}$ ». Ces derniers, pendant leur besogne, commentent tour à tour ce qu'ils font :

«Voici que sur la vergue s'étaient posés des oiseaux de mer.

- Un oiseleur expérimenté les toucha avec une claie de roseaux... et eux, empêtrés

dans ces baguettes engluées, tombaient

- ... entre nos mains.

- La brise emportait leur duvet voltigeant...

- ... et l'écume légère, sur les flots, faisait tourbillonner les plumes. »

11 C'est la reprise exacte d'une description faite par le héros du Satiricon, tandis qu'il se trouve sur un navire. Blutch la transforme en «chœur». Ce sont ces descriptions du Satiricon qui ont surtout retenu les modernes, davantage que les mille rebondissements qui devaient mieux plaire aux contemporains ${ }^{16}$. Il y a le mouvement descendant des oiseaux se posant sur la vergue, puis tombant, puis le mouvement horizontal de leurs plumes gagnant la mer, se fondant avec l'écume; il y a ces oiseaux pris dans la glue et, antithétique, le duvet rebelle qui tourbillonne; il y a ces mots précieux et rares, du moins dans la traduction, comme "vergue », " oiseleurs », "claie de roseaux»; il y a enfin cet emploi de l'imparfait au lieu du parfait qui produit un effet de tableau. La description prosaïque devient naturellement chant à plusieurs voix.

d. Des décalages énonciatifs. Dans cette même planche, Blutch transpose la description du récit au discours (selon la terminologie d'Émile Benveniste). Les matelots commentent leurs propres actions. Ils semblent bien faire ce qu'ils disent: on voit la vergue, les oiseaux, la claie, les plumes. En même temps, ils semblent chanter ce que d'autres ont fait auparavant : c'est un «chœur ", on repère un joueur d'aulos, double flûte grecque, et la description est écrite à la troisième personne et à l'imparfait. La scène est impossible. L'auteur introduit une indécision absolue entre moment du chant et moment de l'action, entre passé et présent, entre spectateurs et acteurs de l'action. Ce dispositif situe Blutch du côté d'une subversion postmoderne de l'énonciation.

e. Des décalages de sens. Le héros et son "petit frère" rencontrent un homme qui leur explique que la ville où ils arrivent a été rasée par des légions romaines en raison de son accointance avec les pirates; ce dernier conclut: «Vous allez pénétrer dans une ville qui ressemble à une campagne ravagée par la peste : vous n'y trouverez rien que cadavres en train d'être dévorés et corbeaux en train de les dévorer ${ }^{17}$. " La planche suivante montre en effet les protagonistes arrivant au milieu d'une multitude de 
crucifiés, puis longeant d'épais charniers humains, le tout accompagné d'un vol de corbeaux affamés. Dans le Satiricon, Encolpe et ses compagnons arrivent de même devant une ville. Un homme leur dit que celle-ci est séparée en deux sortes d'habitants : des testateurs et des captateurs d'héritage. Il emploie ensuite la phrase que nous venons de citer ${ }^{18}$. La mention des cadavres et des corbeaux n'est en fait qu'une image chez Pétrone : elle développe une comparaison avec la ville ravagée par la peste; les corbeaux sont les captateurs d'héritage et les cadavres les testateurs défunts. Blutch, repassant du sens figuré au sens propre, réalise l'image de Pétrone, la prend au mot, ce qui lui fait imaginer une ville ravagée par les Romains. C'est la métaphore elle-même qui crée l'histoire. C'est à partir d'une image de Pétrone, faite en passant, que Blutch construit la suite de l'intrigue.

14 Nous avons relevé des passages avec des intertextes précis, repris tels quels. Nous avons fait l'expérience de ce que Michel Riffaterre appelle l'agrammaticalité: des scènes se comprennent difficilement si l'on ne passe pas par une lecture intertextuelle. Mais il faut dire que le lecteur n'a pas besoin de lire la bande dessinée avec le Satiricon dans l'autre main pour éprouver les effets qu'elle tend à provoquer. Ce qui compte, c'est moins l'élucidation des agrammaticalités que le sentiment d'agrammaticalité luimême. Le lecteur est sans cesse confronté à une énonciation étrange, artificielle, qui bat en brèche le réalisme.

15 Si Blutch ne respecte pas la lettre du roman, il en respecte l'esprit, en ce que, justement, il n'imite pas le Satiricon mais le déplace, comme le Satiricon déplaçait, entre autres, l'Odyssée. On peut parler chez Pétrone de déplacement parodique : réécriture de l'épopée avec un sujet et un style bas. Chez Blutch, il y a au contraire comme un déplacement anti-parodique, qui n'est pas non plus retour à l'épique, mais qui cherche moins à faire rire qu'à poétiser l'univers. Blutch trahit Pétrone comme Fellini l'avait trahi au cinéma... pour mieux rester fidèle à lui. Quelle fidélité ? Fidélité à la satura, comme mélange des récits, des genres, des styles et des registres, et comme satire du monde contemporain : Péplum représente un monde autre, où les figures du Père et de l'autorité n'existent plus, où des sexualités alternatives viennent mettre à bas le modèle hétérosexuel dominant, un monde très éloigné de ce genre du péplum auquel le titre renvoie dans une démarche de distanciation critique. Blutch disait ainsi de son intertexte: "Le Satiricon est complètement obscur, fantomatique, fourmillant, convulsif, brutal... et inachevé, avec des ellipses, des queues de poisson... Ce bouquin est aux antipodes d'une reconstitution hollywoodienne de l'Antiquité. C'est une sorte d'ovni littéraire qui vient de la quatrième dimension. Et c'est ce livre qui m'a dicté l'esprit et le style de la bande dessinée ${ }^{19}$."

16 Pour conclure, nous voudrions nous justifier de n'avoir pas parlé, dans l'étude d'une bande dessinée, de ce qui semble être son élément principal: le dessin. Il est de tradition de dire que la bande dessinée, c'est d'abord de l'image, le texte n'étant que secondaire. On entend la même chose à propos du cinéma. Nous avons voulu montrer ici que le texte pouvait avoir une place capitale dans la facture d'une bande dessinée, même si l'extraordinaire dessin de Blutch n'est pas à négliger. 


\section{NOTES}

1. Voir Paul GRAVETT, Graphic Novels : Everything You Need To Know, New York, Collins Design, 2005.

2. Citons les séries Les Olives noires, 2001-2003, et Socrate le demi-chien, 2002, ainsi que l'illustration du Banquet de Platon chez Bréal, 2002.

3. Entretien de Philippe Dumez avec Blutch, paru dans Jade, 15, 1998 ; consultable sur Internet : www.pastis.org/Blutch/entretienjade.htm (dernière consultation : juillet 2007).

4. Voir le même entretien.

5. SHAKESPEARE, Jules César, III, 1, v. 31-76.

6. Voir Harold вLоом, The Anxiety of influence, a theory of poetry, second edition, New York, Oxford university press, 1997, p. 19-48.

7. Florence DUPONT et Thierry ELOI, L'érotisme masculin dans la Rome antique, Paris, Belin, «L'Antiquité au présent », 2001, p. 327.

8. Entretien de Philippe Dumez avec Blutch, op. cit.

9. Bernard MAGNÉ, Perecollages, 1981-1988, Toulouse, Presses universitaires du Mirail, «Les Cahiers de littératures », 1989, p. 74.

10. BLuTCH, Péplum, Paris, Cornélius, 1996, planche 12. Nous indiquerons dorénavant le numéro des planches.

11. Pétrone, Satiricon, dans Romans grecs et latins, textes présentés, traduits et annotés par

P. Grimal, Paris, Gallimard, « La Pléiade », 1958, p. 46-47.

12. Pétrone, Satiricon, 126, p. 119.

13. BLUTCH, Péplum, 58.

14. Pétrone, Satiricon, 85, p. 73.

15. BLUTCH, Péplum, 64.

16. Voir l'introduction d'O. SERS dans Pétrone, Satiricon, texte établi par A. Ernout, amendé, traduit et commenté par O. Sers, Paris, Les Belles Lettres, «Classiques en poche », 2001, p. X.

17. BLuTch, Péplum, 52.

18. Pétrone, Satiricon, 116, p. 107.

19. Entretien d'Hugues Dayez avec Blutch, dans La nouvelle bande dessinée, Bruxelles, Niffle, «Profession », 2002, p. 44.

\section{AUTEUR}

\section{VIVIEN BESSIÈRES}

Allocataire-Moniteur

Université Toulouse II - Le Mirail

vivienbessieres@yahoo.fr 